\title{
EARLY RESISTANCE CHANGE AND STRESS/ELECTROMIGRATION MODELING IN ALUMINUM INTERCONNECTS
}

\author{
V. PETRESCU*, A.J. MOUTHAAN*, W. SCHOENMAKER ${ }^{*}$ \\ *University of Twente, MESA Research Institute, PO Box 217, 7500 AE Enschede, The \\ Netherlands, \\ "IMEC, ASP-TCAD Division, Leuven, Belgium
}

\begin{abstract}
A complete description for early resistance change and two dimensional simulation of mechanical stress evolution in confined $\mathrm{Al}$ interconnects, related to the electromigration, is given in this paper. The model, combines the stress/ vacancy concentration evolution with the early resistance change of the Al line, that could be [1] a fast technique for prediction of the MTF of a line compared to the conventional (accelerated) tests.
\end{abstract}

(C) 1997 Elsevier Science Ltd

\section{INTRODUCTION}

A serious reliability concern in the last decade for IC manufacturers is electromigration, defined as mass transport resulting from the momentum transfer between the conducting electrons and lattice atoms. The measurement of small resistance changes in the early stages of electromigration has gained considerable interest in the last few years, a reasonable amount of data being available [2,3]. The contradictory behaviour of increase of resistance with time made very difficult to capture all effects in one model. The observations that have been done show that resistance increases linearly with time $[1,2]$ or sometimes an incubation time before the resistance change is noticed [4]. The present paper extends the existing models $[5,6,7,8]$ correlating the mechanical stress/vacancy concentration with early resistance changes of the $\mathrm{Al}$ lines using a two dimensional simulator based on finite element method.

It is generally assumed that there is a linear relation between a change in vacancy concentration and small resistance changes due to electromigration. In our model electromigration flow is related with mechanical stressmigration that opposes to the electromigration flow. Consider a near-bamboo interconnect line, (the line consists of a successive alternation of polycrystalline regions and single crystal regions as indicated in Figure 1) the material flow is due to diffusion of atoms along grainboundaries. A depletion of atoms at the cathode end of a polycrystalline region (usually called cluster) and a mass accumulation at the anode end will give rise to a mechanical stress $\sigma$. Where a large tensile stress develops, a void can be initiated to grow (nucleate) and a hillock can cause cracking of the passivation layer at places where compressive stresses are built up. In a real metallization line, grain boundaries and line imperfections such as dislocations provide suitable diffusion 
paths for atoms. The flux of atoms is modeled, using an opposite but equal flux of vacancies, supposing that atoms diffuse via a vacancy mechanism.

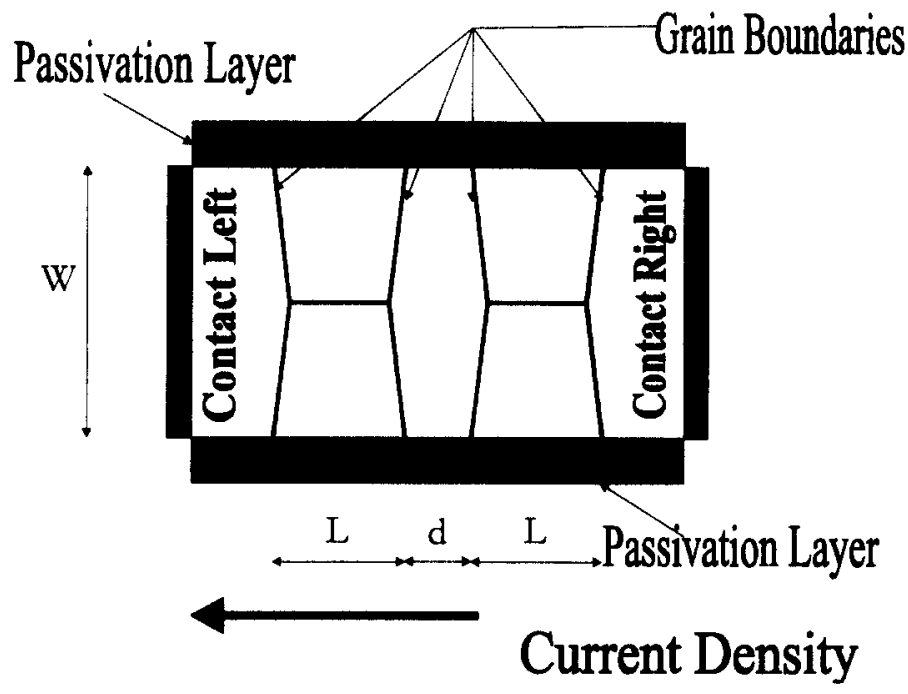

Figure 1 Near-bamboo interconnect line

\section{MODEL DESCRIPTION}

The vacancy flux contains an electromigration induced term and a backflux term induced by a concentration gradient:

$$
J_{V}=-D_{V} \nabla c_{V}+\frac{D_{V}^{c} V_{V}}{k T} Z_{V}^{*} e E
$$

where $c_{V}$ is the vacancy concentration, $Z_{V}{ }^{*}$ is the effective valence, $E$ the electric field which is related to the electric current, $\mathrm{E}=\rho \mathrm{j}$, with $\rho$ the line resistivity. The diffusivity $\mathrm{D}_{\mathrm{v}}$ of vacancies is:

$$
D_{V}=D_{o} \exp \left(\frac{-E_{a}}{k T}\right)
$$

where the activation energy $E_{a}$ has different values in bulk and at grain boundaries The conservation law for vacancies:

$$
\frac{\partial c_{V}}{\partial t}+\nabla J_{V}=G
$$

introduces a sink/source term $\mathrm{G}$ that allows for the creation or annihilation of vacancies at sites as grainboundaries, dislocation lines, surfaces. A vacancy can be eliminated by dislocation climb. For a metal line, encapsulated in a strong passivation, the local change of the volume due to vacancies annihilated or created is the origin of hydrostatic stress. Assuming a finite life time $\left(\tau_{s}\right)$ of a vacancy in the presence of a source/sink $(20 \mathrm{~ms})$ a generation/recombination term for an excess concentration of vacancies compared to the equilibrium value can be introduced [7]:

$$
G=-\frac{c_{\nu}-c_{V_{e}}}{\tau_{S}}
$$


$\mathrm{C}_{\mathrm{Ve}}$ is concentration of vacancies in equilibrium with mechanical stress:

$$
c_{V e}=c_{V o} \exp \left(\frac{\sigma \Omega}{k T}\right)
$$

where $c_{\mathrm{V} 0}$ is the vacancy concentration in the absence of any stress effects, $\sigma$ the mechanical stress, $\Omega$ the atomic volume. The change in the volume of the crystal due to creation/formation of vacancies conforms to Hooke's law, stress:

$$
\frac{d V}{V}=\frac{d \sigma}{B}=\Omega\left(c_{V}-c_{V_{e}}\right)
$$

where $\mathrm{V}$ is an appropriate volume element, $\mathrm{B}$ bulk modulus and $\Omega$ is the activation volume for vacancy generation or vacancy volume itself. The stress evolution in time follows from the derivative of (6):

$$
\frac{\partial \sigma}{\partial t}=B \Omega \frac{c_{V}-c_{V e}}{\tau_{s}}
$$

The model also relates the resistivity of the metal line to vacancy concentration:

$$
\rho=\rho_{0}\left[1+\xi\left(c_{V}-c_{V 0}\right)\right]
$$

where:

$$
\xi=\frac{1}{N_{a}-c_{V}}
$$

and $\mathrm{N}_{\mathrm{a}}$ is the atomic concentration. There are two important assumptions in the model that lack as yet physical proof. One is that the vacancy concentration correlates with resistance change. It has been argued that not the change in vacancy concentration causes $G$ to change, but a straightforward piezoresistive effect is responsible [9]. Second, it is assumed here that the law of Hooke is the 'force' restoring the vacancy concentration, $c_{V}$, back to its local equilibrium $\mathrm{c}_{\mathrm{ve}}$. A physical proof here is also lacking.

\section{SIMULATION RESULTS}

Simulated structures consist on a metal stripe with a near-bamboo microstructure as indicated in fig.1. The metallisation lines have been stressed with a current density of $1 \mathrm{MA} / \mathrm{cm}^{2}$, at $\mathrm{T}=200^{\circ} \mathrm{C}$. The activation energy for vacancies diffusion is $\mathrm{E}_{\mathrm{abulk}}=1.1 \mathrm{eV}$ in bulk and $\mathrm{E}_{\mathrm{agb}}=0.6$ $\mathrm{eV}$ for grain boundary. Other relevant parameters in the model, assuming an aluminum line: $\mathrm{B}=50 \mathrm{GPa}, \mathrm{Z}_{\mathrm{V}}{ }^{*}=20, \tau_{\mathrm{s}}=20 \mathrm{~ms}, \rho_{0}=5 \mu \Omega \mathrm{cm}$. Simulation results show the relative resistance change (Figure 2) and maximum stress evolution (Figure 3) for the structure illustrated in Figure 1 during 1000 hours of current stress. The stress distribution (Figures 4 and 5) across the simulated lines proves the role of a $2 \mathrm{D}$ simulator tool for a complete characterisation of the metal line compared to 1D simulator [10]. The simulated structure takes into account also the cluster interaction [10]. The discontinuity in the slope of the linear increase of the resistance in time can be correlated to different regimes. We can distinguish in cluster interactions: an initial, non interactive regime (Figure 4) and at longer times, the interaction regime, when the 
stress profiles of the clusters overlap (Figure 5). The erratic behaviour of resistance change can also be seen in measurement.

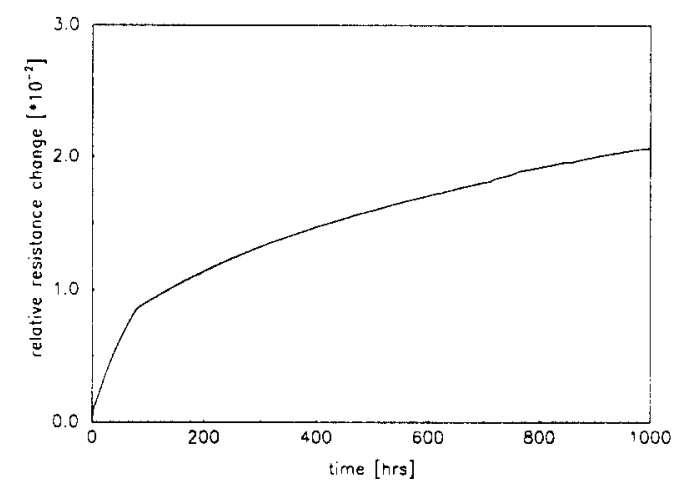

Figure 2 Relative resistance change in time for 1000 hours of current stress for a near-bamboo interconnect line

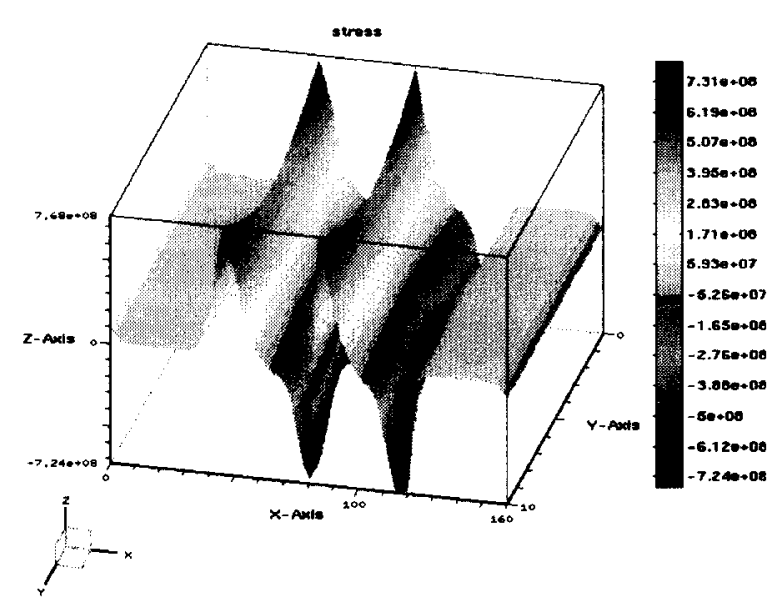

Figure 4 Mechanical stress distribution [in Pa] after 900 hours of current stress

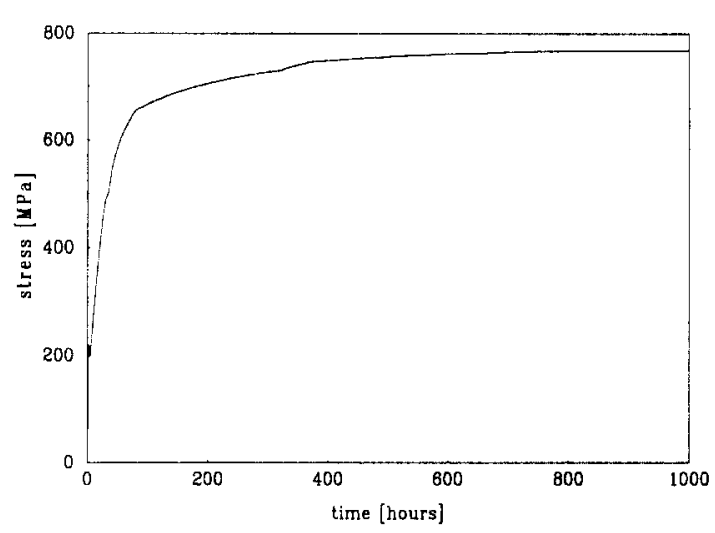

Figure 3 The maximum stress evolution for 1000 hours of current stress for a near-bamboo interconnect line

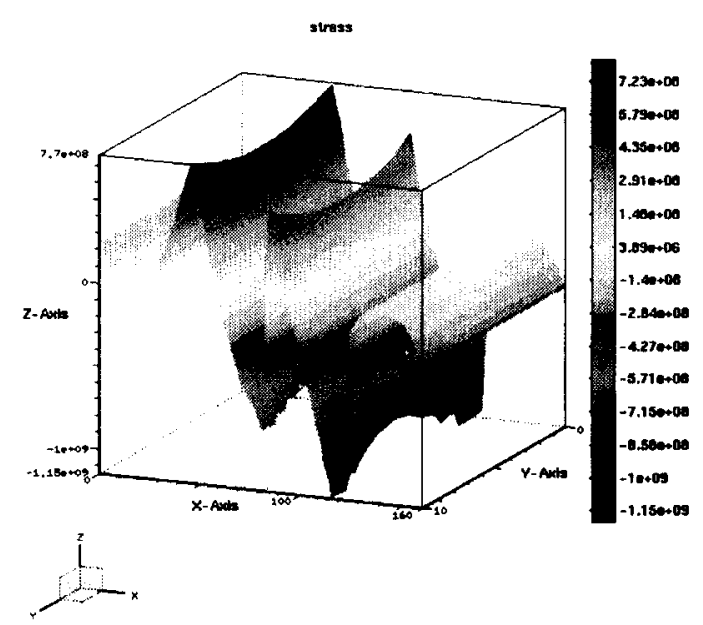

Figure 5 Mechanical stress distribution [in $\mathrm{Pa}$ ] after 1000 hours of current stress

\section{References}

1. J. Niehof, P.A. Flinn, T.J. Maloney, QRE Int. 9, 295, (1993).

2. A. Scorzoni, G.C. Cardinali, G.L. Baldini, G. Soncini, Microelectron Reliab., 30, no.1, 123, (1990).

3. G.L. Baldini, A. Scorzoni and F. Tamarri, Microelectron. Reliab., 33, no. 11/12, 1841, (1993).

4. W. De Ceuninck, J. Manca, V. D'Haeger, L. De Schepper.and L.M. Stals, Proc. ESREF 95, 177, (1995).

5. J. Niehof, H.C. de Graaff, A.J. Mouthaan and J.F. Verwey, Solid St. Electr., 38, no. 10, 1817, (1995).

6. M. Shatzkes and J.R. Lloyd, J. Appl. Phys. 59, 3890 (1986).

7. R. Kirchheim, Acta Mettall. Mater. 40, 309 (1992).

8. M. A. Korhonen, P. Borgesen, K. N. Tu, and C.-Y. Li, J. Appl. Phys. 73, 3790 (1993).

9. A. Scorzoni, I. De Munari, H. Stulens and V. D'Haeger, MRSS Proc. 391, 513, (1995).

10.B. D. Knowlton, J. J. Clement, R. I. Frank and C. V. Thompson, MRSS Proc. 391, 189, (1995). 